\title{
Integration of the hydrogen-storage purification and hydrogen network
}

\author{
Kaiyu Li, Leihao Zhou, Guilian Liu*
}

(School of Chemical Engineering \& Technology, Xi'an Jiaotong University, Xi'an 710049,Shaanxi, China)

For the third iteration of Case 2, the purified product purity, the PFP and initial hydrogen recovery $\left(R_{0}\right)$ are taken as $0.9999,0.45$ and 0.6837 respectively, and the specific optimization process are illustrated below.

\section{Identification of the optimal PFFR}

In the hydrogen network, no hydrogen source's purity is larger than the purified product purity. Sources $\mathrm{SR}_{1}, \mathrm{SR}_{2}, \mathrm{SR}_{3}, \mathrm{SR}_{4}, \mathrm{SR}_{5}, \mathrm{SR}_{6}, \mathrm{SR}_{7}$ and $\mathrm{SR}_{8}$ lie between the PPP and the PFP; their quantitative relationships are obtained according to Eq. (3). $\mathrm{SR}_{9}$ and $\mathrm{SR}_{10}$ lie below the PFP, and their quantitative relationships are obtained according to Eq. (2). The obtained relationships are shown by Eqs. (S1)-(S10). According to the sum of HUS and $F_{w}$ should not excess the tail gas flow rate without considered purification, Eq. (S11) is obtained to limit the feasible PFFR and HUS. ${ }^{8}$ Based on these equations, the quantitative relationship diagram between HUS and PFFR is plotted in Fig S1, and Eq. (S11) corresponding the line CBD. The feasible region is the that surround by OABC. From this figure, it can be seen that the HUS increases along the polyline OAB and the HUS decreases along the line BC. Point B corresponds the maximum HUS, $33.65 \mathrm{~mol} \cdot \mathrm{s}^{-1}$, and the corresponding PFFR is $156.80 \mathrm{~mol} \cdot \mathrm{s}^{-1}$

$$
\begin{aligned}
& F_{p u r}=2.752 \Delta_{s} F_{u, S R_{1}}-109.583 \\
& F_{p u r}=2.819 \Delta_{s} F_{u, S R_{2}}-110.139
\end{aligned}
$$

* Corresponding author: Prof. Guilian Liu, E-mail: guilianliui@ mail.xjtu.edu.cn. 


$$
\begin{gathered}
F_{p u r}=2.926 \Delta_{s} F_{u, S R_{3}}-75.599 \\
F_{p u r}=3.011 \Delta_{s} F_{u, S R_{4}}-40.579 \\
F_{p u r}=3.081 \Delta_{s} F_{u, S R_{5}}-20.959 \\
F_{p u r}=3.103 \Delta_{s} F_{u, S R_{6}}-11.599 \\
F_{p u r}=3.112 \Delta_{s} F_{u, S R_{7}}-17.152 \\
F_{p u r}=3.132 \Delta_{s} F_{u, S R_{8}} \\
F_{p u r}=4.969 \Delta_{s} F_{u, S R_{9}}-31.390 \\
F_{p u r}=6.302 \Delta_{s} F_{u, S R_{10}}-55.216 \\
F_{p u r} \leq-3.250 \Delta_{s} F_{u, i}+266.152
\end{gathered}
$$

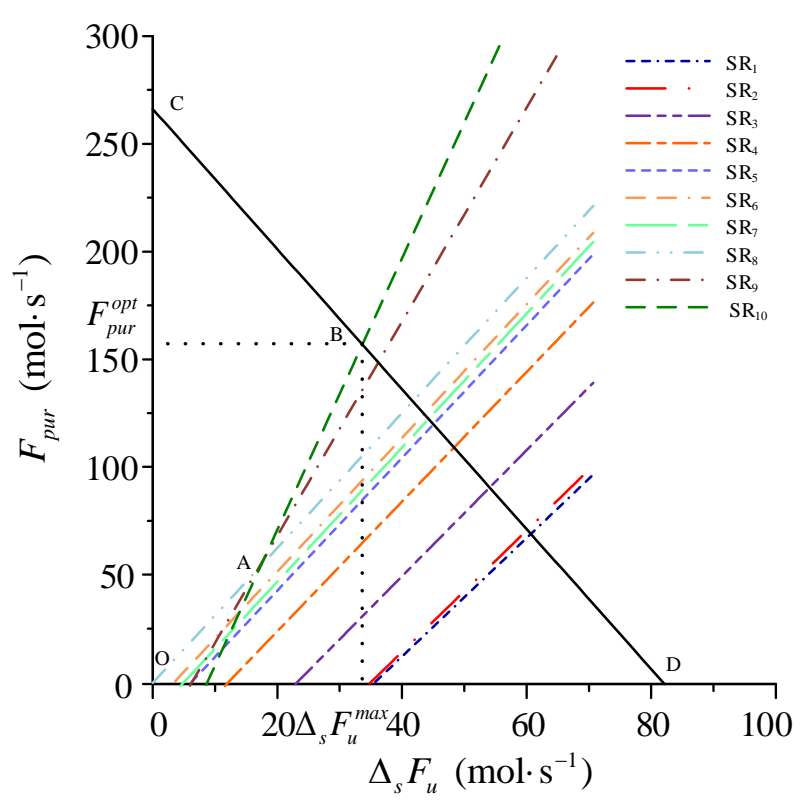

Fig. S1 The quantitative relationship diagram between HUS and PFFR (Case 2)

\section{Identification of the optimal PFP}

With the absorption pressure, the conversion of absorption and temperature taken as $7.5 \times 10^{5} \mathrm{~Pa}$ 0.9999 and $293 \mathrm{~K}$, respectively, the $X$ versus t curves is plotted at each hydrogen source purity according to Eq. (4) and Eq. (7), as shown by Fig. S2. The reaction time increase along the PFP. The 
absorption time $\left(t_{a b s}\right)$ and the weight of the required hydrogen storage material $\left(m_{\text {LaNi }_{5}}\right)$ are calculated by Eq. (10) and Eq. (11), and the result is shown in Table S1. It can be seen that the weight of required hydrogen storage material increases along the PFP.

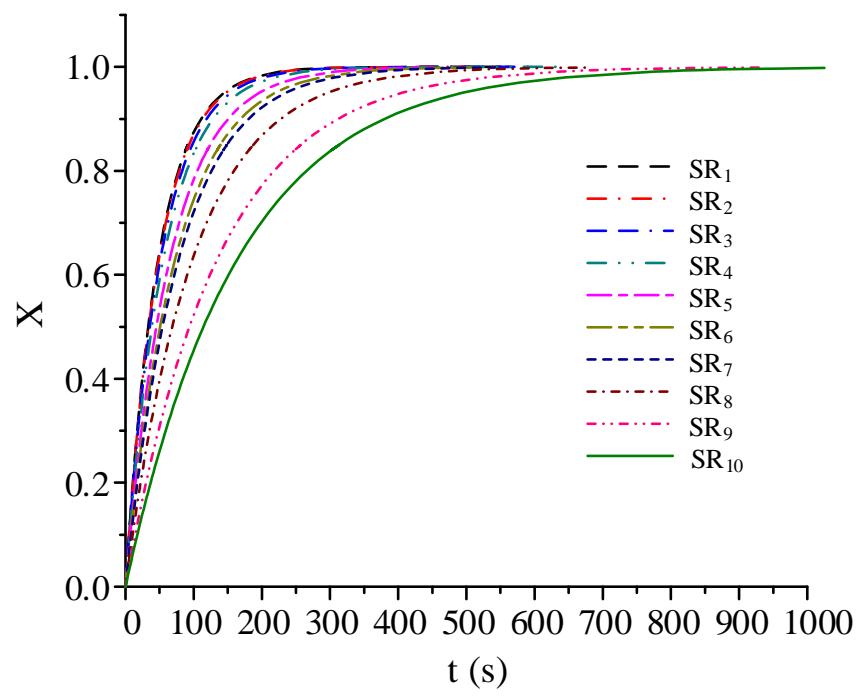

Fig. S2 $X$ versus $t$ curve of LaNis alloy at different purification feed purities (Case 2)

Table S1 tabs and $m_{\text {LaNi }_{5}}$ at different PFPs

\begin{tabular}{ccccccccccc}
\hline Resource & $\mathrm{SR}_{1}$ & $\mathrm{SR}_{2}$ & $\mathrm{SR}_{3}$ & $\mathrm{SR}_{4}$ & $\mathrm{SR}_{5}$ & $\mathrm{SR}_{6}$ & $\mathrm{SR}_{7}$ & $\mathrm{SR}_{8}$ & $\mathrm{SR}_{9}$ & $\mathrm{SR}_{10}$ \\
\hline$c_{\text {pur }}(\mathrm{mol} \%)$ & 87 & 85 & 80 & 73 & 62 & 56 & 53 & 45 & 38 & 35 \\
$t_{\text {abs }}(\mathrm{s})$ & 221.88 & 225.97 & 237.36 & 256.93 & 301.22 & 337.47 & 360.98 & 455.19 & 723.26 & 859.70 \\
$m_{\text {LaNi }_{5}}(\mathrm{~kg})$ & 2256.9 & 2245.6 & 2220.1 & 2192.8 & 2183.5 & 2209.5 & 2236.8 & 2394.8 & 3185.8 & 3576.6 \\
& & & & & & & & & & \\
\hline
\end{tabular}

The quantitative relationships between PFP and HUS are derived based on Eqs. (1)-(3) and shown by Eqs. (S12)-(S21). Based on these equations, the quantitative relationship diagram is plotted in Fig. S3. This figure shows that the HUS change along the polyline OAB, and the pinch lies at the intersection point of $\mathrm{SR}_{10}$ and sink-tie-line. At point $\mathrm{A}$, the HUS reaches the maximum, $43.32 \mathrm{~mol} \cdot \mathrm{s}^{-}$ 1, and the optimal PFP of hydrogen network is 0.385 . According to Eq. (12), the $U$ versus PFP curve is plotted in Fig. 19. It can be seen that the maximum $U$ is achieved at PFP is 0.45 . In the hydrogen network, the optimal PFP is 0.45 , and the corresponding maximum HUS is $33.64 \mathrm{~mol} \cdot \mathrm{s}^{-1}$, accounts 
for $44.69 \%$ of the minimum hydrogen utility consumption of the system without purification.

$$
\begin{aligned}
& \Delta_{s} F_{u, S R_{1}}= \begin{cases}1279.99-1298.79 c_{p u r} & c_{p u r}>0.87 \\
39.82+126.70 c_{p u r} & c_{p u r} \leq 0.87\end{cases} \\
& \Delta_{s} F_{u, S R_{2}}= \begin{cases}1064.33-1082.47 c_{p u r} & c_{p u r}>0.85 \\
39.08+123.70 c_{p u r} & c_{p u r} \leq 0.85\end{cases} \\
& \Delta_{s} F_{u, S R_{3}}= \begin{cases}722.74-752.01 c_{p u r} & c_{p u r}>0.80 \\
25.83+119.12 c_{p u r} & c_{p u r} \leq 0.80\end{cases} \\
& \Delta_{s} F_{u, S R_{4}}= \begin{cases}471.35-511.43 c_{p u r} & c_{p u r}>0.73 \\
13.48+115.78 c_{p u r} & c_{p u r} \leq 0.73\end{cases} \\
& \Delta_{s} F_{u, S R_{5}}= \begin{cases}276.86-322.40 c_{p u r} & c_{p u r}>0.62 \\
6.81+113.16 c_{p u r} & c_{p u r} \leq 0.62\end{cases} \\
& \Delta_{s} F_{u, S R_{10}}= \begin{cases}95.87-138.28 c_{p u r} & c_{p u r}>0.35 \\
8.76+110.61 c_{p u r} & c_{p u r} \leq 0.35\end{cases} \\
& \Delta_{s} F_{u, S R_{8}} F_{u, S R_{6}}= \begin{cases}212.81-261.03 c_{p u r} & c_{p u r}>0.56 \\
3.74+112.31 c_{p u r} & c_{p u r} \leq 0.56 \\
111.25 c_{p u r} & c_{p u r} \leq 0.45 \\
5.32+110.78 c_{p u r} & c_{p u r}>0.38\end{cases} \\
& \begin{array}{ll}
195.63-150.56 c_{p u r} & c_{p u r}>0.53
\end{array}
\end{aligned}
$$




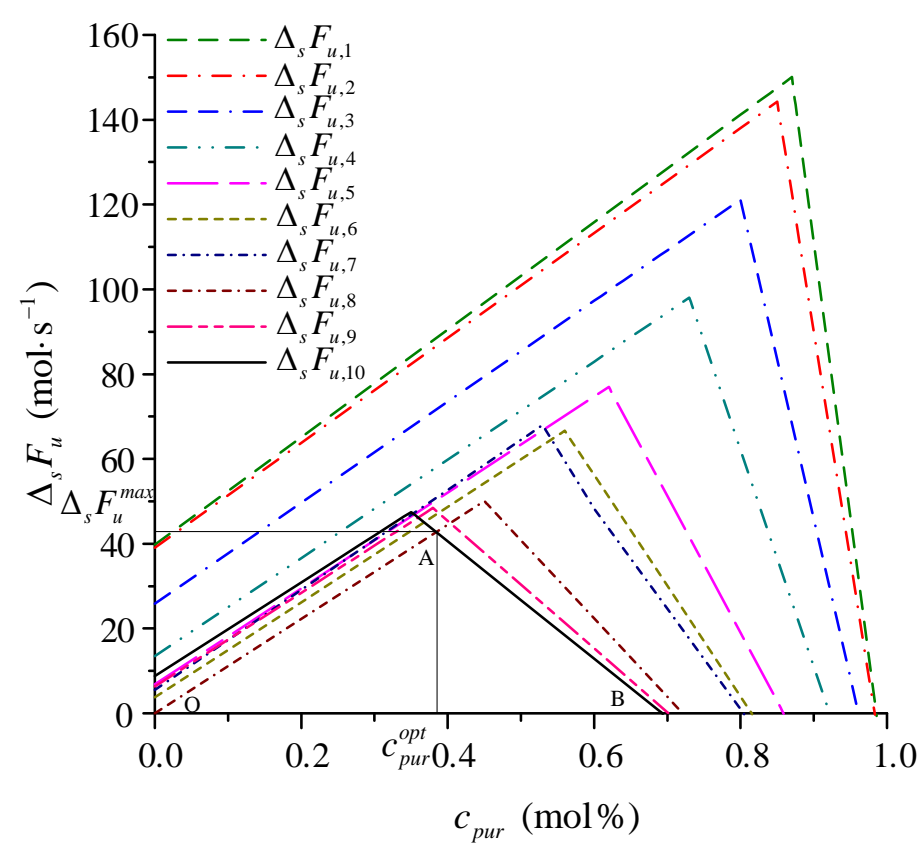

Fig. S3 $\Delta_{s} F_{u}$ versus $c_{\text {pur }}$ diagram of Case 2

\section{Identification of the optimal hydrogen recovery}

According to Eq. (15), the relationship between $m_{\text {LaNi }_{5}}$ and the hydrogen recovery $(R)$ is plotted in Fig. 20, and the optimal hydrogen recovery is identified to be 0.6837098 , and its relative error to that of the previous iteration is less than $1 \times 10^{-3}$.

\section{The hydrogen-storage based purification unit}

The optimal PFFR, PFP and hydrogen recovery are 156.80, 0.45 and 0.6837 , respectively. According to the optimal parameters, the hydrogen storage unit is designed and shown in Table S2. The continuous model of two hydrogen-storage purification units is shown by Fig. 21(a). According to Eq. (17) and Table S1, the desorption and absorption time are $332.84 \mathrm{~s}$ and $455.19 \mathrm{~s}$. Based on the desorption and absorption time, the time distribution of hydrogen-storage based purification unit is plotted and shown by Fig. 21(b). The hydrogen network of Case 2 with the minimum hydrogen 
consumption (or maximum HUS) is shown by Fig. S4. Similar with Case 1, the cost of the material at different prices is compared with that of PSA, as shown by Fig. 22. In this figure, the cost of PSA is calculated according to Eq. (29), ${ }^{41}$ and two lines intersect at point A, which corresponds the price 918.63 USD $\mathrm{kg}^{-1}$. The hydrogen storage purification is superior to the PSA when the price of LaNi 5 is less than this value.

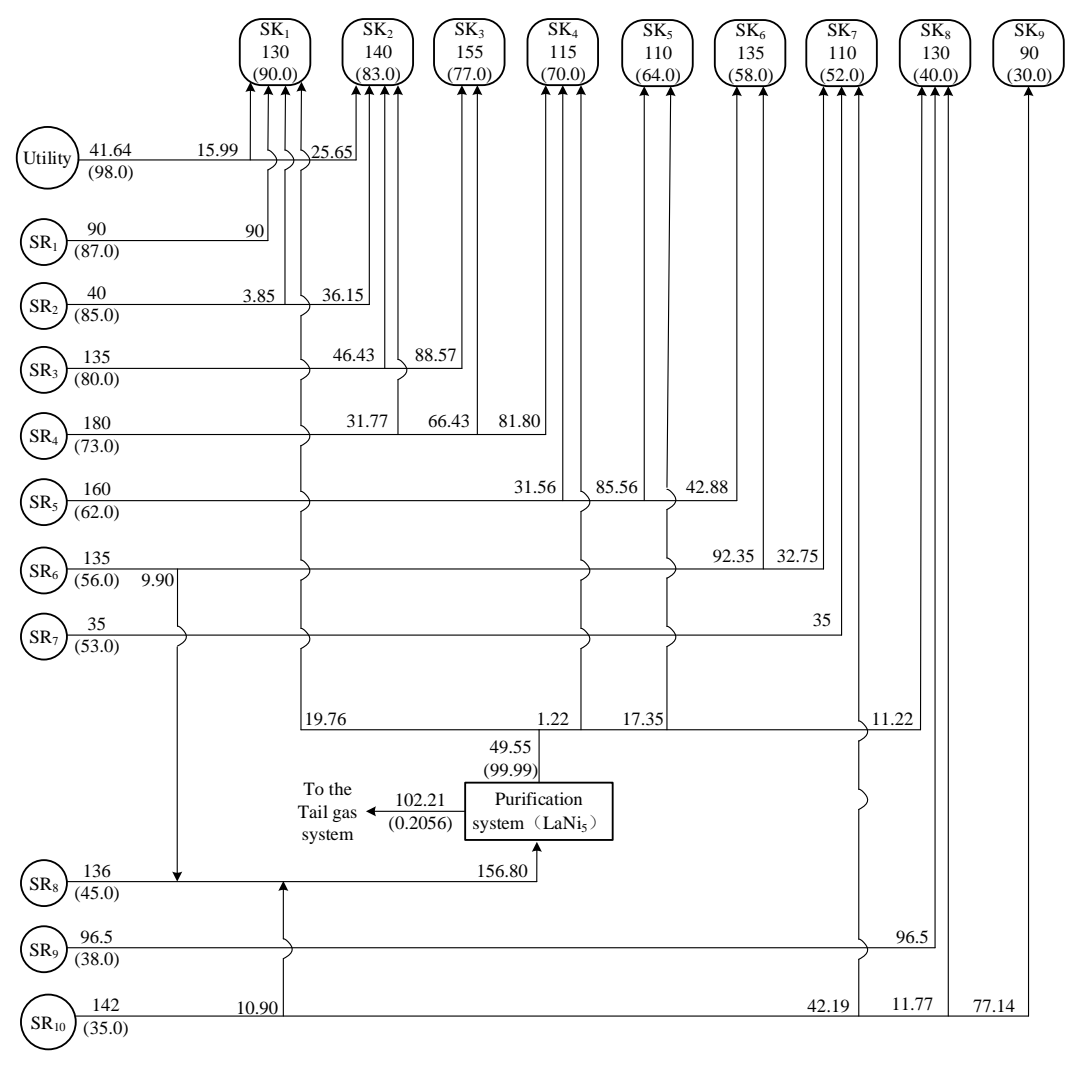

Fig. S4 The configuration of the hydrogen network based on the optimal hydrogen-storage purification unit (Case 2) 